Cinémas

Revue d'études cinématographiques

Journal of Film Studies

\title{
L'importance des processus cognitifs et de la recherche empirique en études cinématographiques
}

\section{Paul S. Cowen}

Volume 12, numéro 2, hiver 2002

Cinéma et cognition

URI : https://id.erudit.org/iderudit/024879ar

DOI : https://doi.org/10.7202/024879ar

Aller au sommaire du numéro

Éditeur(s)

Cinémas

ISSN

1181-6945 (imprimé)

1705-6500 (numérique)

Découvrir la revue

Citer cet article

Cowen, P. S. (2002). L'importance des processus cognitifs et de la recherche empirique en études cinématographiques. Cinémas, 12(2), 39-59.

https://doi.org/10.7202/024879ar
Résumé de l'article

Dans la mesure où toute théorie du cinéma compose, implicitement ou explicitement, avec le rôle du spectateur, l'auteur avance qu'il faut postuler l'activité de processus psychologiques - conscients ou inconscients - qui se révèlent finalement d'ordre cognitif. Il fait notamment valoir que ceux qui critiquent la théorie cognitive du cinéma semblent parfois ne pas comprendre ou ne pas connaître les courants qui caractérisent actuellement la psychologie cognitive et la cognition sociale. Présentant un éventail de recherches expérimentales récentes, dont plusieurs sont menées au laboratoire qu'il dirige, l'auteur montre comment l'empirisme permet d'expliciter plusieurs notions introduites en études cinématographiques par Bordwell. Mais surtout, les données qu'il présente font la preuve que la recherche empirique peut contribuer pour beaucoup au développement de la théorie du cinéma. 


\title{
L'importance des processus cognitifs et de la recherche empirique en études cinématographiques
}

\section{Paul S. Cowen}

\begin{abstract}
RÉSUMÉ
Dans la mesure où toute théorie du cinéma compose, implicitement ou explicitement, avec le rôle du spectateur, l'auteur avance qu'il faut postuler l'activité de processus psychologiques - conscients ou inconscients qui se révèlent finalement d'ordre cognitif. Il fait notamment valoir que ceux qui critiquent la théorie cognitive du cinéma semblent parfois ne pas comprendre ou ne pas connaitre les courants qui caractérisent actuellement la psychologie cognitive et la cognition sociale. Présentant un éventail de recherches expérimentales récentes, dont plusieurs sont menées au laboratoire qu'il dirige, l'auteur montre comment l'empirisme permet d'expliciter plusieurs notions introduites en études cinématographiques par Bordwell. Mais surtout, les données qu'il présente font la preuve que la recherche empirique peut contribuer pour beaucoup au développement de la théorie du cinéma.
\end{abstract}

\section{ABSTRACT}

Since all film theories are implicitly or explicitly concerned with spectatorship, the author proposes that this requires postulating the activity of conscious and unconscious psychological processes that are ultimately "cognitive" in nature. He then puts forward that criticism of cognitive film theory often stems from a misunderstanding or lack of knowledge about current trends in cogni- 
tive and social-cognitive psychology. A brief survey of research that is pertinent to several of Bordwell's ideas includes experiments conducted in the author's laboratory and demonstrates the contribution that empirical studies can make to film theory, especially with regard to the importance of narrative structure, the relations between cognition and emotions and the role of cognitive biases in film interpretation by both spectators and critics.

\section{La cognition, les émotions et l'empirisme}

Il n'est pratiquement aucune théorie du cinéma qui ait pu faire l'impasse sur l'activité du spectateur et sur les processus psychologiques qu'elle implique. C'est que le cinéma a la capacité, non seulement de structurer le temps et l'espace de manière à produire une réalité ressemblant au monde accessible à la perception naturelle, mais encore de susciter des réactions affectives intenses. Le cinéma semble donc présupposer ces processus. Du coup, il semble obliger le théoricien à en tenir compte quand vient le temps d'expliquer la fonction expressive des films ainsi que leur influence sur les plans psychologique, social et politique. Si bien que toutes les théories du cinéma, celles où la question du spectateur apparaît en creux aussi bien que celles qui l'abordent de manière explicite, se devaient de traiter d'un certain nombre de processus psychologiques, comme le relève Stam (2000, p. 229). Et, de fait, aussi contradictoires qu'apparaissent entre elles les approches critiques du cinéma, toutes se sont développées à partir des travaux les plus divers menés en psychologie et en psychiatrie, et toutes prêtent des propriétés d'ordre psychologique aux spectateurs (ou aux réalisateurs).

Ainsi, la position filmo-linguistique d'Eisenstein (1949, p. 28-44; p. 108-121) ou sa théorie du montage (p. 72-83), la doctrine réaliste de Bazin (1967) et l'approche féministepsychanalytique (Mulvey, 1989) sont autant de perspectives qui impliquent une théorie de la psychologie du spectateur. Cela vaut aussi pour la proposition moins typique de Schatz (cité dans Bordwell, 1996, p. 5) selon laquelle le cinéma fonctionne comme le mythe du fait que la narration permet la conciliation des contradictions culturelles, car ce processus profond suppose 
encore une activité cognitive. À la limite, quand des tenants de la théorie du positionnement du sujet avancent que la subjectivité est une transformation du système de représentation, sur la base de quoi ills déclarent qu'il n'y a pas lieu de tenir compte de données empiriques, il faut bien leur répondre que cette transformation suppose des processus cognitifs, à moins qu'ils ne croient que le phénomène se produise comme par magie. Par contre, comme le signale Bordwell (1996, p. 10), les tenants de la critique culturelle sont plus près d'une proposition claire et précise concernant des processus psychologiques lorsqu'ils affirment que les institutions sociales imposent aux individus des catégories et des structures de connaissance.

Bref, le cinéma suppose des effets chez le spectateur. Ce sont précisément ces effets qui permettent au théoricien de relier son interprétation d'un film à la compréhension de l'œuvre par un auditoire. En effet, le théoricien se comporte au cinéma comme monsieur ou madame Tout-le-monde: c'est par le biais de présuppositions, d'heuristiques et de schémas inconscients que chacun comprend un film, l'évalue et communique ce qu'il a vu. Mais, à la différence du simple spectateur, le théoricien tend à projeter sa propre perspective interprétative sur l'inconscient d'un spectateur anonyme produit par voie de généralisation. Il s'attache alors à décrire ce processus par lequel tous sont censés comprendre et apprécier un film, processus ayant donc valeur de condition sine qua non.

Ironiquement, deux des premières théories du cinéma furent élaborées par des psychologues concentrant leurs efforts sur les processus de perception et de cognition, qu'ils mettaient en relation avec des émotions et des réponses esthétiques. Mais l'orientation gestaltiste de Münsterberg ([1915] 1970) et d'Arnheim (1957) serait beaucoup moins populaire que les approches d'inspiration freudienne, ces dernières disposant de concepts autrement plus dynamiques et dramatiques. Le problème avec ces approches, c'est que certaines de leurs hypothèses sur le fonctionnement de l'inconscient étaient tenues pour des faits avérés. Les choses ont un peu changé avec l'émergence d'une critique d'inspiration cognitive. Mais, comme le remarque Prince (1996, p. 74-75), plusieurs sont restés convaincus de la 
validité de leurs propositions bien qu'elles échappent à toute possibilité de vérification. Et parmi les théoriciens qui n'ont pas ignoré des données pertinentes, plusieurs les ont mal comprises. Enfin, comme Bordwell l'a affirmé (1989, p. 18-21), un certain nombre de théoriciens et de critiques considèrent que la validité de leur théorie de prédilection réside essentiellement dans le fait qu'elle s'applique à des films particuliers, ce dont ils font la démonstration à grand renfort d'arguments rhétoriques et tendancieux.

À une telle méthode de validation, on peut toujours objecter des arguments scientifiques et rationnels. Mais certaines découvertes sont plus décisives. La recherche en cognition sociale (Fiske et Taylor, 1991, p. 346-406) a mis au jour de nombreuses formes de préjugés régissant la perception et le jugement, et ce, dans les situations les plus courantes de la vie quotidienne. Et comme ces partis pris cognitifs participent d'un sens commun, il est à peu près impossible de les détecter: tout un chacun est convaincu de sa sagesse, de la véracité de sa perception et de son interprétation des faits. Même le théoricien est aux prises avec ces a priori qui offrent l'illusion d'une impression claire, ou d'une décision appropriée à une configuration de stimuli qui n'annonce pas explicitement sa catégorie d'appartenance ni son sens ultérieur. Étant donné que les messages cinématographiques sont véhiculés en bonne partie par le déploiement de données visuelles et spatiales, par le comportement non verbal des protagonistes et par des relations spatiotemporelles créées par le montage, ils sont plus ambigus ou équivoques que les messages verbaux et, en conséquence, ils sont plus susceptibles de déclencher des heuristiques et des schémas peu objectifs à la réception.

La résistance à la théorie cognitive du cinéma semble parfois résulter d'une mauvaise compréhension de ses approches, d'une méconnaissance de la méthode empirique et d'une représentation erronée des processus cognitifs. Par exemple, Stam (2000, p. 241) insiste sur le fait que l'approche cognitive reste incapable d'expliquer la diversité des interprétations et des réponses esthétiques suscitées par un seul et même film. Pas plus qu'elle ne peut expliquer pourquoi Cruising (Friedkin, 1980) induit des 
réactions homophobiques, pourquoi The Seige (Zwick, 1998) induit des réactions anti-Arabes ou pourquoi certains spectateurs ont des réactions misogynes en visionnant Fatal Attraction (Lyne, 1987). Pour formuler de telles objections, il faut ne pas avoir pris connaissance de certains textes (Cowen, 1991 - par exemple) passant en revue les nombreuses recherches effectuées en cognition sociale ainsi que leurs développements théoriques, travaux qui témoignent de ce que l'approche cognitive est peutêtre celle qui peut le mieux traiter les questions que Stam lui reproche de laisser sans réponse.

L'approche cognitive n'exclut pas les hypothèses selon lesquelles des facteurs psychosexuels ou idéologiques influent sur le spectateur. Seulement, les caractéristiques du contenu, du style ou de la structure des films qui ont pour effet d'attirer ou de repousser le spectateur doivent être précisées en fonction des caractéristiques, conscientes ou non, du répertoire de ce dernier. C'est dans la mesure où des facteurs causaux sont ainsi précisés qu'on peut étudier les effets s'y rattachant. En effet, il n'est pas suffisant de postuler que les besoins ou motivations à l'origine de la réalisation d'un film ou à l'origine des identifications des spectateurs sont universels, qu'ils s'appliquent à tous les films et à tous les spectateurs ou presque. Une théorie fondée sur des universaux ne pourra jamais fournir l'explication qu'exige Stam quant à la diversité des réactions, des interprétations, des appréciations et des critiques du même film.

Pour réfuter la critique cognitive, il ne suffit pas non plus de fabriquer un homme de paille cognitif. Il ne suffit pas de faire valoir que la cognition et les émotions sont des processus opposés, et de surenchérir en affirmant de manière simpliste que la cognition se résume à un mécanisme mental d'inférence, de déduction et de froid calcul. Reprocher à la critique cognitive de dissocier artificiellement la pensée et les émotions, c'est passer sous silence plus de quarante ans de travaux portant sur la théorie socio-cognitive des émotions et sur les liens entre émotions et processus inconscients (pour un résumé de ces travaux, voir Fiske et Taylor, 1991, p. 409-461; pour des applications de ces travaux au cinéma, voir Tan, 1996). Donner de la cognition l'image caricaturale d'une calculatrice ou d'un ordinateur, 
c'est ignorer l'ensemble des recherches portant sur les présuppositions conscientes ou inconscientes qui influent sur notre interprétation de la réalité sociale et physique. Le cognitivisme " computationnel " pourrait caractériser l'orientation de certains fondateurs de ce qui allait devenir la science cognitive, mais cinquante ans plus tard, cette perspective restreinte ne correspond plus à la grande variété de phénomènes cognitifs étudiés par diverses disciplines. Finalement, même si on affirme $a$ priori que les processus émotionnels et inconscients du spectateur ne sont pas de la compétence des approches cognitivistes, il n'en est pas moins illogique de conclure que certaines des propositions impossibles à vérifier, provenant des camps freudien ou lacanien aussi bien que de la sémiotique, sont valides simplement parce qu'elles s'orientent vers les émotions plutôt que vers la pensée. Et même quand des recherches révèlent les insuffisances de certaines explications d'ordre cognitif, cela ne suffit pas à rendre valides les approches dont cherche à se démarquer la critique cognitive.

Les sciences sociales, la recherche empirique, les méthodes scientifiques, la narratologie, même la logique, à l'instar d'autres systèmes de croyances, doivent parfois être soumis à la critique. Certes, le scepticisme envers ces entreprises ne se manifeste pas toujours au moyen d'une solide critique d'ordre philosophique. Mais, comme le fait remarquer un théoricien du cinéma qui est aussi psychanalyste (Greenberg, 1993, p. 5 et 12), le mélange particulier des théories de Freud, de Marx et de la sémiotique qui a dominé la théorie du cinéma pendant une certaine période est plus radical et orthodoxe dans son appui à la doctrine de Freud que n'importe quelle tendance ou pratique contemporaine en psychologie ou en psychiatrie. Peut-être parce qu'il est plus facile de maintenir une attitude stricte et orthodoxe quand on s'épargne l'effort d'appliquer une théorie à des personnes réelles, évitant du coup leurs protestations. Prince (1996, p. 74) a montré comment l'adhésion aux principes freudiens et lacaniens, entre autres, repose sur la fausse croyance que ces propositions théoriques sont déjà bien vérifiées empiriquement.

Étant donné l'impossibilité de vérifier les hypothèses concernant des universaux - ces derniers relèvent davantage de la 
métaphysique -, l'approche cognitive dont les écrits de Bordwell (1989 et 1997) sont représentatifs et l'orientation générale de la psychologie expérimentale depuis quarante ans préferent des théories et des modèles mieux circonscrits qui s'appliquent à des collections de données plus restreintes, même si en général les processus cognitifs ont leur propre universalité. À la recherche des principes universels, on préfere celle des invariances locales (un contexte, une activité, une culture, etc.). Cela suffit peut-être à décourager d'autres théoriciens d'approfondir le contenu ou les observations concrètes de l'approche cognitive. Le fait que les méthodes empiriques les plus fines tombent généralement hors du cadre de la formation en études cinématographiques représente un autre obstacle à cet égard. Par contre, en abandonnant les explications reposant sur des motivations et des besoins universels, la psychologie a pu mieux reconnaître et respecter l'hétérogénéité de diverses populations, y compris celles qui ne participent pas de la prétendue idéologie dominante.

Bref, étant donné que la majorité des théories du cinéma impliquent un spectateur, et qu'elles ont d'ailleurs été développées par des théoriciens à partir de leur propre activité spectatorielle, il est impossible d'ignorer des théories et des données concernant la perception, la cognition (les processus inconscients, les pensées, les jugements, les évaluations, les interprétations, la mémoire, etc.), les émotions et la communication sociale.

\section{Mes deux formations}

Mon orientation et mes méthodes s'inscrivent dans le droit fil des suggestions de Bordwell (1996, p. 29) : servi par une double formation en psychologie expérimentale et en études cinématographiques, j'étudie des questions locales plutôt que globales, et j'ai souvent tenté de créer des rapports entre la recherche empirique et des variables diverses provenant d'horizons différents. Lorsque j'ai effectué mes premières recherches portant sur le cinéma, je n'avais pas encore entrepris mes études dans ce domaine. Quelques années plus tard, à mi-parcours de ma formation, je me suis rendu compte que mes intuitions et hypothèses initiales, pour naïves qu'elles avaient été, s'accordaient le mieux 
avec les notions développées par des théoriciens rejetant ou ignorant le rôle des processus psychologiques, le raisonnement logique et les méthodes scientifiques. C'est que ma fréquentation du cinéma européen des années cinquante et soixante m'avait convaincu que les structures narratives standardisées du cinéma américain, c'est-à-dire hollywoodien, entravaient le spectateur, l'empêchant d'attribuer à des motivations, à des actions ou à des événements connus autre chose que des significations convenues ou de découvrir entre eux des relations nouvelles. Cette intuition ne pouvait qu'être renforcée par ma connaissance émergente de cette critique considérant la forme narrative comme la maîtresse de l'idéologie dominante, comme une prostituée séduisant des clients-spectateurs pour le compte de son patron. C'est que dans cette perspective, telle que la résume Aumont (1992, chap. 5), le cinéma narratif nous trompe: il cache le contrôle qu'il exerce sur notre perception, il nous enchaîne à l'idéologie dominante en donnant à son discours la forme d'une histoire, il nous incite à croire que les événements présentés par le film se sont déroulés naturellement au lieu d'être déterminés par un auteur, un producteur, un metteur en scène, des comédiens, des techniciens, etc.

\section{Recherches sur l'utilisation du schéma narratif}

Il me semblait donc raisonnable de prévoir que les films à la structure non narrative, et surtout ceux dont la structure est franchement dysnarrative, libéreraient le spectateur de la prison idéologique dans laquelle les films narratifs contribuent insidieusement à l'enfermer, qu'ils lui permettraient d'arriver à une interprétation non tendancieuse. À l'époque, je ne voyais aucune contradiction entre cette prévision naïve et mon orientation cognitive en psychologie. D'ailleurs, mes recherches antérieures (Cowen, 1984) m'avaient déjà permis de constater que des courts métrages présentant des actions et des événements de sens opposés induisent des interprétations plus véridiques, c'està-dire moins tendancieuses, que les versions textuelles de la même information contradictoire. Néanmoins, mes premières recherches portant directement sur les structures moins traditionnelles allaient indiquer que je n'avais pas tout à fait raison de 
croire qu'un auditoire traiterait une forme non narrative avec enthousiasme.

À partir d'une courte histoire ayant un certain degré d'ambiguïté mais plutôt linéaire, on a généré trois autres versions en variant l'agencement des scènes. Deux de ces nouvelles versions étaient quasi linéaires et la troisième, non linéaire. Dans un premier temps, les étudiants en psychologie des arts ont indiqué que les quatre versions suscitaient des émotions fort différentes. Cette observation globale allait aussi être valable pour les données plus précises obtenues auprès d'un échantillon plus important de spectateurs dont chacun n'avait vu qu'une seule version. Ce sont ces données qui intéressent particulièrement mon propos. Les spectateurs qui ont visionné la version quasi linéaire la plus éloignée de la version d'origine ont eu un taux de rappel équivalent à celui observé chez les spectateurs de la version originale linéaire, mais $40 \%$ d'entre eux ont donné de l'histoire une interprétation ne s'accordant pas avec l'histoire de la version originale. Les données confirmaient l'idée de Burch (1981, p. 12) selon laquelle l'activité cognitive permet au spectateur de rapporter rétroactivement l'un à l'autre des plans qui sont temporellement éloignés dans le film. Dans des versions où le rapport entre les plans est moins évident, moins de spectateurs arrivent à reconstruire l'histoire originale. C'est qu'une légère modification apportée au montage suffit pour que l'on prête aux protagonistes des intentions et des motivations très différentes, voire opposées, la nature de l'intrigue variant en conséquence. Et lorsque les spectateurs ne perçoivent pas de rapports explicites, ils traitent l'ordre des scènes comme une représentation du véritable ordre chronologique des événements et ils tentent de rendre cet ordre cohérent en produisant une interprétation qui explique cette séquence de scènes. Cette tentative était évidente chez les spectateurs de la version non linéaire, où il était presque impossible de percevoir un rapport spatial ou causal entre les scènes. Mais leur tentative échouant, les spectateurs ont jugé que cette dernière version n'avait pas d'histoire, et leur taux de rappel fut très faible. Par ailleurs, les jugements portés sur les deux protagonistes ont varié considérablement selon l'ordre des plans. Les spectateurs de la version non linéaire, même s'ils étaient loin 
d'avoir apprécié cette version, n'ont pas hésité à juger les protagonistes de façon stéréotypée, et ce, malgré qu'ils n'aient pu comprendre quelque intrigue que ce soit et qu'ils n'aient pu se rappeler les actions et les événements.

Ainsi, les spectateurs n'ont pas manifesté quelque désir que ce soit d'abandonner leur schéma narratif malgré l'incohérence de certaines scènes ou du film entier. Je ne mattendais pas à ce que soit si forte la tendance à utiliser un schéma narratif pour rendre cohérente une série d'expériences visuelles. Mes recherches antérieures ayant indiqué que les films suscitent davantage d'interprétations véridiques que les textes, j'étais loin de prévoir des résultats similaires à ceux obtenus par Bartlett (1932). Dans cette recherche devenue exemplaire, des lecteurs d'une histoire dont le contenu et la structure leur étaient étrangers ont inconsciemment déformé le texte en oubliant certains faits, en en fabriquant d'autres et en réorganisant le matériel pour accommoder l'histoire à l'état de leurs connaissances et à leur répertoire de présuppositions. Enfin, il semble que la tendance à utiliser un schéma narratif est généralisée, qu'un préjugé narratif est inhérent à notre traitement de la succession temporelle des stimuli internes ou externes. S'il est un candidat qui puisse prétendre à l'universalité, il semble bien que ce soit le schéma narratif. Cette hypothèse coïncide avec l'observation du fait que les histoires ont toujours existé, que dans toutes les sociétés humaines connues circulent des histoires qui organisent et expliquent les expériences personnelles, sociales et culturelles (Branigan, 1992, p. 1). Le schéma narratif est possiblement une structure profonde, au même titre que celle mise au jour par Chomsky (1965) pour expliquer l'apparition et l'acquisition des langues naturelles chez tous les humains. De fait, Carroll (1980) a analysé la grammaire du cinéma à la lumière des contributions de ce linguiste. Dans une tentative plus profonde, Grodal (1997a et 1997b) propose que l'universalité et l'apriorisme des processus mentaux associés aux films narratifs canoniques (et à d'autres films) reposent sur la nécessité de leur développement dans une perspective évolutionniste et sur leur rôle fonctionnel comme processus centraux génériques reliant les perceptions, cognitions, émotions et réactions involontaires aux actions volontaires. 
Le fait que les interprétations et les identifications different d'avec l'agencement des scènes supporte indirectement l'idée que des processus inconscients sont à l'œuvre, ainsi que le postule la théorie du positionnement du sujet. Les données s'accordent également avec l'idée d'une personnification ou d'une identification fondée sur des traits formels. La focalisation sur l'un des protagonistes avant que l'autre ne fasse son apparition en serait un exemple. Selon Bordwell (1989, p. 161), le schéma qui permet cette personnification ou identification est une variété du schéma de la personne, fréquemment utilisé pour interpréter des événements sociaux. En ce qui concerne l'interprétation d'un récit quasi linéaire ou non linéaire, ce type de schéma peut fonctionner à deux niveaux. Au premier niveau, la personnification du style de montage quasi linéaire ou non linéaire amène le spectateur à imaginer que le thème du film concerne les changements d'avis, l'indécision, l'influence du hasard et de l'imprévu, etc. Au second niveau, l'identification avec l'un ou l'autre des protagonistes, ainsi que son appréciation, dépend également de l'ordre des scènes. Étant donné que les comportements ne sont jamais isolés de leur contexte et que le contexte influe sur l'interprétation d'une action donnée, un changement dans l'agencement des scènes modifie en conséquence le contexte dans lequel cette action apparaît.

On a vu qu'une version non linéaire, avec la difficulté de compréhension qu'elle comporte et l'appréciation négative qu'elle suscite, n'empêche pas les spectateurs de juger les protagonistes en recourant aux stéréotypes disponibles dans leurs schémas des personnes ou des groupes. En général, un film qui s'écarte beaucoup des attentes du spectateur est déplaisant et suscite des jugements esthétiques défavorables. Cette attitude négative se manifeste également chez des spectateurs qui réussissent à découvrir et à reconstruire la linéarité de l'histoire originale. Un bon nombre de recherches en cognition ont démontré depuis longternps que les gens préferent appliquer et confirmer des schémas existants plutôt que de faire l'expérience de l'incertitude et de l'ambiguïté, normalement accompagnées d'émotions négatives et déstabilisantes. Cette tendance reflète le désir d'économiser les ressources et les efforts cognitifs. Les 
spectateurs utilisent donc des heuristiques qui les ont bien servis antérieurement au lieu de mettre en question la validité de leurs schémas ou de développer de nouveaux schémas pour mieux interpréter des stimuli atypiques.

Une autre recherche effectuée dans mon laboratoire (Roberts, Cowen et MacDonald, 1996) a permis de comparer les réponses suscitées par des versions linéaire, quasi linéaire et non linéaire d'un moyen métrage dramatique. À une réduction de la linéarité correspondait une plus faible compréhension de l'histoire, surtout pour les scènes où se manifestaient des émotions négatives. C'est qu'il était difficile de cerner des rapports causaux entre ces scènes et d'autres événements de l'histoire. En conséquence, à une linéarité moins marquée correspond un plus faible rappel de l'information visuelle ainsi qu'une appréciation moins favorable du film, de l'histoire et des protagonistes. Quant à la version non linéaire, elle était particulièrement déplaisante parce que la résolution du conflit impliquant un protagoniste aimé était divulguée avant que ne soient connus les obstacles qu'il avait dû surmonter pour atteindre son but. L'évaluation négative de cette version s'accorde avec les postulats et les données de Zillman (1980) concernant le suspense et le plaisir. Ses recherches ont montré que la connaissance à peu près certaine de l'issue des conflits réduit significativement les réactions physiologiques et le plaisir du spectateur. Au contraire, si le film fait persister le doute quant à l'issue des conflits, l'intérêt pour les protagonistes et le suspense généré sont maximisés, et la satisfaction éprouvée ultérieurement est d'autant plus grande. Les facteurs d'empathie et de probabilités subjectives ont donc une influence considérable sur les réactions affectives et la compréhension du drame, mais ils ne figurent pas dans un grand nombre de théories du cinéma.

\section{D'autres schémas importants}

La prépondérance des schémas narratifs, utilisés de façon quasi automatique, n'exclut pas que d'autres schémas soient activés ou que les spectateurs soient sensibles à d'autres paramètres des œuvres cinématographiques. Bordwell (1989) identifie plusieurs schémas utilisés dans l'interprétation des films, dont le 
schéma général des catégories, qui se précise selon les genres de films et de discours. Lors d'une série d'études récentes dans mon laboratoire, Pouliot (2001) a comparé des schémas associés aux grandes catégories que sont le film narratif de fiction et le film documentaire, qui généralement se distinguent l'un de l'autre par leurs traits formels, leurs structures, les types de liens causaux qu'ils établissent ou l'usage qu'ils font de la rhétorique (Nichols, 1981).

Des films narratifs et documentaires présentant les mêmes thèmes et ayant les mêmes valences émotionnelles ont été comparés du point de vue de leur effet sur divers types de spectateur. Tel que prévu, les films narratifs ont eu un effet plus marqué que leur contrepartie documentaire, mais cette tendance était plus prononcée pour les spectateurs utilisant une stratégie visuelle dans l'intégration de l'information visuelle et verbale. Une stratégie verbale d'intégration, par contre, produit un effet semblable pour les deux types de discours. Fidèles à leur mission, les films narratifs ont incité les spectateurs à accorder beaucoup plus d'importance à l'information centrale, celle relative à l'intrigue, quà l'information périphérique. Alors que pour les récits documentaires, on a accordé à ces deux niveaux à peu près la même importance. En ce qui concerne la comparaison du point de vue de la valence émotionnelle des films, ceux ayant un thème tragique (le sida et la mort) ont eu un plus grand impact que les films ayant un thème plutôt joyeux (l'amour et le mariage) ou que les films de valence mixte (la biographie de Ghandi).

L'influence du schéma pour un genre particulier en relation avec d'autres caractéristiques structurales a fait l'objet d'une étude empirique de Cowen et Lebel (1998). Durant la première partie d'un film noir, principalement consacrée à l'exposition et à la mise en place de l'intrigue, les attentes des spectateurs reliées à ce genre de film étaient assez grandes : n'étant pas encore suffisamment au fait de l'histoire qui se développerait, ils n'étaient pas en mesure de distinguer de façon non équivoque l'information centrale de l'information périphérique. De sorte qu'ils se sont souvenus de scènes arrivant relativement tôt dans le film, mais qui se révéleraient ultérieurement non essentielles pour 
l'histoire, dans la mesure où elles étaient marquantes et ne suivaient pas les attentes du genre. Mais dès que les spectateurs perçurent plus clairement les contours de l'histoire, les actions et les événements non essentiels au développement ou à la résolution du conflit central ne furent plus gardés en mémoire et le genre n'influa plus sur la manière dont les spectateurs organisaient mentalement le film.

La même étude a également traité de la distinction entre l'intrigue et l'histoire, ainsi que le suggérait Thompson (1988, p. 39). Une des caractéristiques clés des films narratifs est la façon de structurer la divulgation de l'information essentielle à l'histoire. Par cette structuration, le film dirige les réactions émotionnelles et l'intérêt du spectateur vers le développement des conflits et leur résolution ultérieure. Dans cet ordre d'idées, il est possible que le schéma activé pour percevoir la suite continue d'actions et d'événements soit différent du schéma plus abstrait qui établit des liens causaux entre les unités plus larges de l'histoire, les épisodes. Les résultats suggèrent qu'un schéma d'histoire ou un schéma narratif constitue une heuristique moins efficace pour un spectateur encore indécis quant à la nature ou à l'orientation de l'histoire.

La structuration de l'intrigue exerce une influence particulière sur la perception du fil conducteur de l'histoire lorsque le film crée une ambiance de mystère, évoque la tromperie ou multiplie les fausses pistes. Pour ce genre de film, le spectateur préfere commencer avec un schéma d'événements (Wyer et al., 1985), qui est à la fois moins ordonné et plus focalisé sur des données précises et concrètes. Plus tard, c'est grâce à un schéma narratif que le spectateur dirigera son attention et qu'il régira l'organisation mentale du récit. Le modèle de rappel des spectateurs après qu'ils eurent visionné le film de notre étude indique qu'ils ont bien créé une représentation mentale concise s'articulant autour des actions clés. Cette représentation permet une reconstruction détaillée qui sera utilisée s'il faut raconter l'histoire de nouveau, et elle reste disponible dans un futur proche. Par ailleurs, certains des éléments de cette étude permettaient de conclure que le schéma qui correspond à la connaissance de l'histoire n'est pas identique au schéma procédural permettant de raconter l'his- 
toire, lequel correspond plutôt à l'intrigue, c'est-à-dire au déroulement concret suivant un plan de divulgation mesurée.

Malgré l'importance de l'histoire et de la structure narrative, Petric (1983, p. 207) considère qu'en se préoccupant de ces facteurs, surtout dans le cadre d'une analyse sémiologique traditionnelle, on ignnore des éléments variés et complexes qui distinguent et caractérisent le cinéma. On ignore aussi d'autres types de schémas quil reconnaissent et exploitent le rôle d'autres traits formels. Par exemple, l'utilisation d'un schéma de cercles concentriques, ou «bull's-eye» (Bordwell, 1989, p. 170-181), semble tenir à la perception de correspondances synchroniques entre les traits de caractère attribués à un protagoniste, le contexte où l'action se déroule et les caractéristiques formelles du film. Un deuxième schéma interprétatif, plus dynamique, agit comme une heuristique d'expressivité (Bordwell, 1989, p. 181-186). Par son intermédiaire, les contextes et les personnages sont interprétés comme des expressions du sens attribué aux traits formels ou vice versa. Ainsi, l'interprétation du film pourrait se fonder, entre autres, sur la relation entre des protagonistes, sur le contraste ou l'accord entre l'information visuelle et verbale, ou sur le rythme et la vitesse du montage. Au laboratoire, des chercheurs ont tenté d'étudier ces influences et ces interactions.

Une série d'expériences menées par Gagnon (1995) a permis de constater que la relation est moins évidente entre la perception des traits formels, les inférences concernant les protagonistes et leur environnement, et les réactions physiologiques et émotionnelles du spectateur. Les réponses à deux courts métrages, l'un dramatique et l'autre non dramatique, étaient comparées. Les spectateurs ont vu une version de chaque film avec un rythme de montage ou stable ou accéléré. Aucune des versions n'a produit un effet sur le rythme cardiaque des spectateurs, même si le montage était plus rapide. Néanmoins, le contenu du film dramatique (un homme avec un couteau poursuivant une femme dans la rue et se préparant à l'attaquer lorsqu'elle arrive chez elle) a porté les spectateurs à croire qu'ils étaient moins calmes et plus angoissés, à ne pas aimer le film et à inférer que la femme dans l'histoire déteste l'homme (même si elle ne sait pas qu'il est présent). L'accélération du montage influence le 
spectateur seulement s'il a reçu une fausse rétroaction selon laquelle son rythme cardiaque avait accéléré au moment du visionnement. Dans ces conditions, les spectateurs étendent les traits de dynamisme et d'intensité impliqués par le montage aux sentiments éprouvés par les protagonistes. Cependant, cet effet n'arrive pas à influer sur la perception qu'ont les spectateurs de leurs propres réponses émotionnelles, qui étaient semblables indépendamment du type de montage. Le lien entre les traits formels et l'appréciation du film était moins direct et différait pour les hommes et pour les femmes. Les hommes croyant que leur réaction physiologique avait été plus intense ont dit avoir éprouvé un plus grand plaisir en regardant le film dramatique malgré son contenu négatif. Par contre, les femmes étaient comme des tenants de la théorie féministe du cinéma : elles ont beaucoup moins aimé le film dramatique lorsqu'elles ont reçu une fausse rétroaction les informant que leur rythme cardiaque s'était accéléré durant la présentation du film.

Dans d'autres contextes, les spectateurs different non seulement dans leurs réactions émotionnelles et leur évaluation du film, mais encore dans leur façon d'interpréter et d'intégrer l'information verbale et visuelle. Fondamentalement, chacun traite des stimuli en fonction de schémas de soi. Ce type de schéma correspond à des aspects dynamiques et stratégiques du concept de soi qui entrent en jeu lorsqu'il y a interaction entre le soi et des stimuli sociaux, réels ou médiatisés. Raza (1999) a observé l'activité d'un tel schéma. Lorsque des hommes incarcérés regardent un film ambigu quant à l'aspect criminel des agissements du protagoniste, ce dernier est perçu comme un criminel, même si le début du film a clairement laissé entendre le contraire. Et si le même film débute par un prologue indiquant que le protagoniste a déjà été incarcéré mais que l'histoire reste ambiguë à cet égard, il n'y a pas le moindre doute quant à ses intentions criminelles. Des spectateurs n'ayant pas de crime à leur actif, eux, vont généralement livrer une interprétation moins tendancieuse.

Un autre film ambigu quant aux intentions criminelles des protagonistes a été présenté dans le cadre d'une étude portant sur les différences individuelles dans l'utilisation des schémas (Cowen, 1992). Les spectateurs d'orientation visuelle ont dans 
tous les cas une meilleure mémoire de l'information visuelle, qu'elle soit ambiguë ou non. En même temps, leur interprétation des actions et des événements est fortement influencée par un schéma verbal si un message verbal leur est présenté avant le film. Dans ces conditions, ces spectateurs ont une perception prédéterminée et non ambiguë. Par contre, si ces spectateurs d'orientation visuelle reçoivent le message verbal après avoir vu le film, ils résistent à l'influence de cette information claire et maintiennent leur interprétation initiale, plus ambivalente. $\grave{A}$ l'inverse, des spectateurs n'ayant pas d'habileté en imagerie mentale ou en mémoire visuelle ne sont pas influencés par le message offert avant le film, mais s'en servent en grande mesure pour lever l'ambiguité dont le film vient de témoigner. Donc, théoriquement, si ces deux types de spectateurs discutent du même film, ils pourraient se voir en désaccord relativement au sens du film (c'est-à-dire sur le fait qu'a eu lieu ou non un crime), et ce, en raison d'une utilisation différente des schémas verbaux et visuels.

L'influence persistante des schémas préexistants, même quand un film semble avoir de quoi les déjouer, est évidente encore une fois dans les résultats d'une série de recherches dirigées par Dubé (2000). Des spectateurs n'ayant pas d'opinion tranchée sur les casinos ou les jeux de hasard ont regardé deux versions d'un documentaire portant sur ce sujet, une partie des spectateurs ayant droit à une version favorable aux casinos et aux jeux de hasard, une autre à une version défavorable. Dans chacune des versions figuraient des informations dont la valence et le sens étaient soit cohérents soit incohérents par rapport à ce point de vue de départ. Indépendamment du point de vue initial ou de la cohérence entre ce point de vue et l'information donnée ultérieurement, les spectateurs ont été grandement influencés par les informations confirmant le stéréotype social négatif associé aux jeux de hasard, aux casinos et aux joueurs obsessionnels. Par contre, il n'y avait pas de corrélation entre leur attitude personnelle et une information négative et tendancieuse.

Finalement, il faut mentionner l'importance des schémas de groupes dans nos stéréotypes raciaux, culturels, sexuels, etc. Un stéréotype ne se limite pas à la simple perception d'une per- 
sonne en fonction de son appartenance à un groupe donné. Les caractéristiques formelles et concrètes du contexte influent sur l'utilisation et l'efficacité de ces schémas. Dans une revue de la littérature pertinente pour l'étude des stéréotypes au cinéma (1991), j'ai fait cette observation: la plus ou moins grande influence d'un stéréotype sur l'interprétation d'un spectateur dépend, entre autres, de la perception qu'a ce spectateur du rôle causal joué par l'appartenance à un groupe dans le cadre du récit, de sa perception du conflit ou de la coopération entre les groupes dans le récit et, surtout, de sa perception du statut du protagoniste (un étranger, un immigrant, un membre d'un groupe minoritaire, etc.) dans le contexte dépeint. D’ailleurs, plus un individu se démarque de son groupe d'appartenance et plus des stéréotypes tendront à se manifester. D'après les résultats d'une recherche de Roberts et MacDonald (1999), lorsque les intentions criminelles d'un protagoniste asiatique ne sont pas claires, les spectateurs tendent à s'en tenir aux données ambiguës et postulent le doute raisonnable concernant sa culpabilité, tendance qui ne se manifeste pas quand le protagoniste est de race blanche. Ce préjugé positif à l'égard du protagoniste asiatique est le produit de l'ensemble des stéréoypes qui nous font considérer les Asiatiques en général d'un oil favorable.

\section{Un appel à l'interdisciplinarité}

Cette brève présentation de quelques recherches empiriques portant sur le rôle de certains processus cognitifs dans la réception des films visait à démontrer que l'approche cognitive ou la théorie cognitive du cinéma propose plus que de nouvelles orientations théoriques. Si elle se passe de cas de force majeure ou de drames, c'est pour mieux tenir compte du comportement et de l'expérience de spectateurs réels, tout en reconnaissant le rôle primordial des processus inconscients et des émotions. Étant donné ma formation en psychologie expérimentale, en cognition et en études cinématographiques ainsi que mon expérience comme thérapeute, j'ai un esprit éclectique à l'égard des films et des théories, ce que je conçois comme un privilège. Mais cela explique aussi que je ne suis pas toujours à l'aise avec ceux qui insistent sur les méthodes scientifiques, pas plus 
d'ailleurs qu'avec leurs opposants qui, dominés parfois par la rhétorique et les opinions, résistent à toute tentative de validation ou de vérification. Ni la science ni les théories du cinéma ne devraient être considérées comme des icônes ou présentées comme la voie consacrée vers la vérité. Car toute doctrine scientifique, religieuse, idéologique, esthétique, ethnocentrique ou égoïste - si absorbée par soi-même, isolée et ignorante des autres systèmes et de leur point de vue, risque la stérilité, à moins qu'on ne considère comme productif le fait que ses adeptes s'engagent dans des différends qui ne peuvent se régler qu'à coups d'arguments et de luttes aussi bien rhétoriques que physiques. J'espère quant à moi que cet article ne côtoiera pas des textes manifestant cette propension.

\section{Université du Québec à Montréal}

\section{RÉFÉRENCES BIBLIOGRAPHIQUES}

Arnheim, 1957 : R.udolf Arnheim, Film as Art, Berkeley, University of California Press, 1957.

Aumont et al., 1992 : Jacques Aumont et al., Aesthetics of Film, Austin, University of Texas Press, 1992.

Bartlett, 1932: F. C. Bartlett, Remembering: A Study in Experimental and Social Psychology, London, Cambridge University Press, 1932.

Bazin, 1967 : André Bazin, What Is Cinema?, Berkeley, University of California Press, 1967.

Bordwell, 1989: David Borwell, Making Meaning, Cambridge, Harvard University Press, 1989.

Bordwell, 1996: David Bordwell, «Contemporary Film Studies and the Vicissitudes of Grand Theory», dans David Bordwell et Noël Carroll (dir.), Post-Theory, Madison, University of Wisconsin Press, 1996.

Bordwell, 1997 : David Bordwell, On the History of Film Style, Cambridge, Harvard University Press, 1997.

Branigan, 1992: Edward Branigan, Narrative Comprehension and Film, New York, Routledge, 1992.

Burch, 1981 : Noël Burch, Theory of Film Practice, Princeton, Princeton University Press, 1981.

Carroll, 1980 : John M. Carroll, Toward a Structural Psychology of Cinema, The Hague, Mouton, 1980.

Chomsky, 1965: Noam Chomsky, Aspects of the Theory of Syntax, Cambridge, MIT Press, 1965.

Cowen, 1984: Paul S. Cowen, "Film and Text: Order Effects in Recall and Social Inferences ", Educational Communication and Technology Journal, vol. 32, 1984, p. 131-144. 
Cowen, 1988: Paul S. Cowen, "Manipulating Montage: Effects on Film Comprehension, Recall, Person Perception and Aesthetic Responses", Empirical Studies of the Arts, vol. 6, n' 2, 1988, p. 97-115.

Cowen, 1991 : Paul S. Cowen, "A Social-Cognitive Approach to Ethnicity in Films", dans Lester Friedman (dir.), Unspeakable Images, Urbana, University of Illinois Press, 1991.

Cowen, 1992: Paul S. Cowen, "Visual Memory, Verbal Schemas and Film Comprehension ", Empirical Studies in the Arts, vol. 10, n 1, 1992, p. 33-55.

Cowen et Lebel, 1998: Paul S. Cowen et Alain Lebel, "The Influence of Story, Plot and Genre on Memory for Action in a Film", Empirical Studies of the Arts, vol. 16, $\mathrm{n}^{\circ} 1,1998$, p. 71-83.

Dubé, 2000: Gaëtane Dubé, "Analyse du traitement de l'information présentée dans une émission d'information en fonction du point de vue, de la nature et du niveau de cohérence de l'information, de l'attitude et du but" (thèse de doctorat), Montréal, Université du Québec à Montréal, 2000.

Eisenstein, 1949: Sergei Eisenstein, Film Form, New York, Harcourt \& Brace, 1949.

Fiske et Taylor, 1991 : Susan T. Fiske et Shelley E. Taylor, Social Cognition, New York, McGraw-Hill, 1991.

Gagnon, 1995: Alain Gagnon, "L'influence du contenu et de la structure de montage des films sur les attributions émotionnelles consécutives à une information erronée sur l'activité cardiaque" (thèse de doctorat), Montréal, Université du Québec à Montréal, 1995.

Greenberg, 1993: Harvey Roy Greenberg, Screen Memories, New York, Columbia University Press, 1993.

Grodal, 1997a: Torben Grodal, "Emotions, Cognitions and Narrative Patterns in Film ", Proceedings: Symposium on Cognitive Science and the Future of Film Studies, Lawrence, University of Kansas, 1997.

Grodal, 1997b: Torben Grodal, Moving Pictures: A New Theory of Film Genres, Feelings and Cognition, Oxford, Clarendon Press, 1997.

Mulvey, 1989: Laura Mulvey, Visual and Other Pleasures, Bloomington, Indiana University Press, 1989.

Münsterberg, 1970: Hugo Mïnsterberg, Film: A Psychological Study, New York, Dover, [1916] 1970.

Nichols, 1981 : Bill Nichols, Ideology and the Image, Bloomington, Indiana University Press, 1981.

Petric, 1983: Vlada Petric, "Barthes Versus Cinema», Sight \& Sound, vol. 52, n 3, 1983, p. 205-207.

Pouliot, 2001 : Louise Pouliot, "L'influence de la structure et de la valence émotionnelle du récit filmique sur la reconnaissance de l'information" (thèse de doctorat), Montréal, Université du Québec à Montréal, 2001.

Prince, 1996: Stephen Prince, "Psychoanalytic Film Theory and the Problem of the Missing Spectator", dans David Bordwell et Noël Carroll (dir.), Post-Theory, Madison, University of Wisconsin Press, 1996.

Raza, 1999: Elaine Raza, "Le rôle des motivations sociales dans la confirmation d'une attente criminelle. Une application du modèle théorique de Neuberg auprès d'une population criminalisée " (thèse de doctorat), Montréal, Université du Québec à Montréal, 1999.

Roberts, Cowen et MacDonald, 1996: Daniel S. L. Roberts, Paul S. Cowen et Brenda MacDonald, "Effects of Narrative Structure and Emotional Content on 
Cognitive and Evaluative Responses to Film and Text ", Empirical Studies of the Arts, vol. $14, \mathrm{n}^{\circ} 1,1996$, p. $33-47$.

Roberts et MacDonald, 2000 : Daniel S. L. Roberts et Brenda E. MacDonald, "Effects of Protagonist's Race and Salience and Participants'SES on Film/TV Inferences and Perception", Revue internationale de psychologie sociale, tome 13, $\mathrm{n}^{\circ}$ 1, 2000, p. 7-28.

Stam, 2000 : Robert Stam, Film Theory, Malden, Blackwell, 2000.

Tan, 1996: Ed S. Tan, Emotion and the Structure of Narrative Film, Mahwah, Lawrence Erlbaum Associates, 1996.

Thompson, 1985: Kristin Thompson, Breaking the Glass Armor, Princeton, Princeton University Press, 1988.

Wyer Jr et al., 1985: Robert S. Wyer Jr et al., "Event Memory: The Temporal Organization of Social Action Sequences", Journal of Personality and Social Psychology, vol. 49,1985 , p. $855-877$.

Zillman, 1980 : Dorf Zillman, "Anatomy of Suspense», dans P. H. Tannenbaum (dir.), The Entertainment Function of Television, Mahwah, Lawrence Erlbaum Associates, 1980 , p. $133-163$. 